\title{
Cellular pattern formation during Dictyostelium aggregation
}

\author{
Thomas Höfer ${ }^{\text {a }}$, Jonathan A. Sherratt ${ }^{b}$, Philip K. Maini ${ }^{a}$ \\ a Centre for Mathematical Biology, Mathematical Institute, University of Oxford 24-29 St Giles', Oxford OXI 3LB, UK \\ ${ }^{\mathrm{b}}$ Nonlinear Systems Laboratory, Mathematics Institute, University of Warwick, Coventry CV4 7AL, UK
}

Received 1 September 1994; revised 4 February 1995; accepted 16 February 1995

Communicated by M. Mimura

\begin{abstract}
The development of multicellularity in the life cycle of Dictyostelium discoideum provides a paradigm model system for biological pattern formation. Previously, mathematical models have shown how a collective pattern of cell communication by waves of the messenger molecule cyclic adenosine $3^{\prime} 5^{\prime}$-monophosphate (cAMP) arises from excitable local cAMP kinetics and cAMP diffusion. Here we derive a model of the actual cell aggregation process by considering the chemotactic cell response to cAMP and its interplay with the cAMP dynamics. Cell density, which previously has been treated as a spatially homogeneous parameter, is a crucial variable of the aggregation model. We find that the coupled dynamics of cell chemotaxis and cAMP reaction-diffusion lead to the break-up of the initially uniform cell layer and to the formation of the striking cell stream morphology which characterizes the aggregation process in situ. By a combination of stability analysis and two-dimensional simulations of the model equations, we show cell streaming to be the consequence of the growth of a small-amplitude pattern in cell density forced by the large-amplitude cAMP waves, thus representing a novel scenario of spatial patterning in a cell chemotaxis system. The instability mechanism is further analysed by means of an analytic caricature of the model, and the condition for chemotaxis-driven instability is found to be very similar to the one obtained for the standard (non-oscillatory) Keller-Segel system. The growing cell stream pattern feeds back into the cAMP dynamics, which can explain in some detail experimental observations on the time evolution of the cAMP wave pattern, and suggests the characterization of the Dictyostelium aggregation field as a self-organized excitable medium.
\end{abstract}

\section{Introduction}

The amoeboid microorganism Dictyostelium discoideum has long been studied as a paradigm model system for biological pattern formation. Its life cycle shows the transition from solitary amoebae to the formation of a multicellular fruiting body with two specialized cell types. Experimental work has elucidated many of the cellular mechanisms involved in Dictyostelium development, and on this basis a quantitative picture of the dynamics of pattern formation in this species is emerging (e.g. [1] and references therein).

Particular progress has been made in the understanding of the initial stage of multicellular development, involving the aggregation of up to $10^{5}$ amoebae to a mound, from which the fruiting body develops (passing through a motile "slug" stage). Aggregation necessitates the coordination of the activities of cells in a territory several orders of magnitude larger than the micro-environment of a single cell. This is achieved by wave-like 


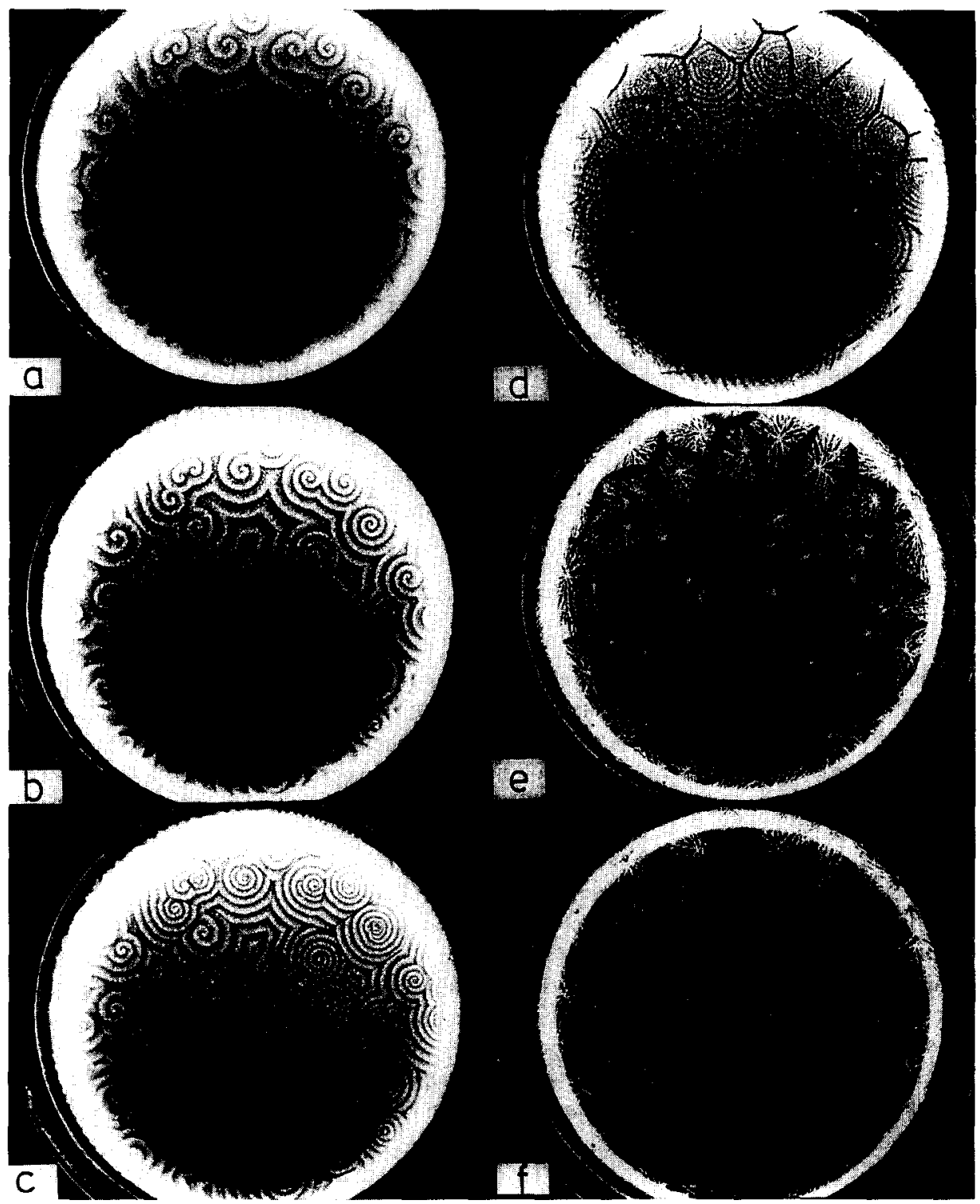

Fig. 1. Aggregation of Dictyostelium (strF NP377) on an agar plate, showing the formation of spiral cAMP waves which induce cell movement (a-c), the onset of cell streaming (d), and the developed cell stream morphology in the whole aggregation territory (e-f). Pictures are taken ca. $10 \mathrm{~min}$ apart; the diameter of the dish is $5 \mathrm{~cm}$. The position of the cAMP waves in (a)-(d) can be inferred from the different light scattering responses of elongated (moving) and rounded (stationary) cells; amoebae elongate under the influence of the cAMP waves and form the bright bands in the photograph. Courtesy of P.C. Newell.

chemical communication via cyclic adenosine $3^{\prime} 5^{\prime}$-monophosphate (cAMP). The kinetics of cAMP production and degradation, spatially coupled by diffusion in the extracellular medium, have been modelled on the basis of detailed experimental data [2,3]. These reaction-diffusion models show that the Dictyostelium aggregation field constitutes an excitable medium with respect to the cAMP dynamics, which supports the collective wave phenomena observed experimentally [4]. Typically, an aggregation territory is organized by a cAMP target wave pattern forced by an autonomously oscillating centre, or a spiral wave of cAMP, which in both cases 
induces periodic movement steps of the cells towards the centre of the pattern

Cells show a directed motile response towards increasing cAMP concentration (positive chemotaxis). Previous mechanistic models of Dictyostelium as an excitable medium have neglected the chemotactic cell movement. This is a reasonable first approximation, as the typical chemotactic cell velocity (ca. $20-30 \mu \mathrm{m} \mathrm{min}^{-1}$ ) is about an order of magnitude smaller than the typical cAMP wave speed (ca. $300 \mu \mathrm{m} \mathrm{min}^{-1}$ ). Thus one can expect that, despite inducing cell movement, the cAMP waves will have negligible effect on the cell density distribution. This is supported by experimental observations [5], and a quantitative argument will be given for this in Section 2 below. This argument, however, holds only for the cell density profile in the direction of cAMP wave propagation, while the actual aggregation process takes place on a two-dimensional spatial domain. Indeed, the experiments indicate that cell movement contributes significantly to the dynamics of aggregation [Figs. 1d-f]. Cells do not simply move straight to the aggregation centre, but form a characteristic pattern of cell streams along which aggregation proceeds after this break-up of the initially homogeneous cell layer. Cell streaming provides a primitive example of morphogenesis in an ensemble of communicating cells. It might have the functional significance of rapidly bringing cells in direct contact, which triggers developmental gene expression [6], and also of increasing the average cell velocity and thus the speed of aggregation. In this paper we are interested in a mechanistic explanation of this phenomenon. Such an explanation must consider both the chemical and motile responses of an individual cell to cAMP. Combining a model of the chemotactic response recently proposed by Höfer et al. [7] on the basis of experimental results with the model equations for the cAMP dynamics [2,8], we have derived an integrated model of aggregation in the cellular ensemble [9]. Here we show by a combination of analytical and numerical approaches that this rather simple model captures in surprising detail the phenomenology of aggregation as observed in situ. The mechanism which generates the cell stream pattern is identified as a chemotaxis-driven instability, forced by the repetitive cAMP waves. On the basis of this model, the Dictyostelium aggregation field can be characterized as a self-organized excitable medium.

The derivation of the aggregation model is outlined in Section 2. Periodic plane wave solutions are obtained close to the wave solutions of the corresponding cAMP signalling model with the cell density "clamped" at a constant value. In Section 3 we analyse the stability of these periodic plane wave solutions in two space dimensions, and demonstrate the existence of an instability which is essentially linked to cell chemotaxis. The instability mechanism is investigated further in Section 4 by means of an analytic caricature of the model, for which an explicit stability criterion can be derived. In Section 5 two-dimensional numerical simulations of the full nonlinear model are presented and compared with experimental observations, followed by a discussion of the results in Section 6.

\section{The model}

The mechanistic models of the cAMP wave dynamics are based on two distinct cellular modes of action of this substance. Extracellular cAMP activates the cAMP-synthesizing machinery of a cell, mediated by binding of cAMP to specific cell surface proteins (cAMP receptors). This in turn activates a cascade of intracellular components, "switching on" the cAMP-synthesizing enzyme (adenylate cyclase). The net effect of this pathway can be modelled by an autocatalytic production term in the conservation equation for extracellular cAMP, associated with a fast time scale $[2,8]$. On the other hand, cAMP also induces a desensitization of this pathway towards further stimulation, on a somewhat slower timescale. Different desensitization mechanisms have been considered (cAMP receptor desensitization [2,8], calcium-mediated desensitization of adenylate cyclase [3], G-protein mediated desensitization [10]). A minimal model of the activation/desensitization dynamics (derived 


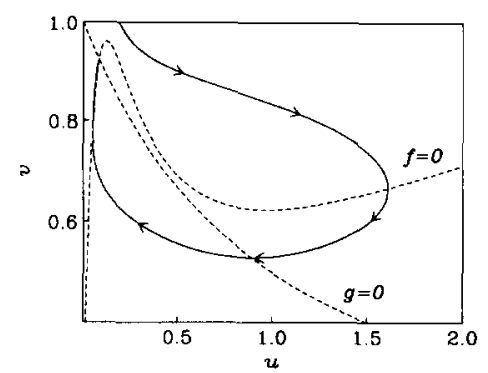

Fig. 2. Phase plane of the local kinetics of system (1),(2), with typical "excitation" trajectory (solid line) and nullclines (dashed lines). Parameters as in Table 2.

from experimental evidence on receptor interconversion as a main desensitization step, but not limited to this mechanism) reads, in nondimensionalised form [2],

$$
\begin{aligned}
& \frac{\partial u}{\partial t}=\sigma\left[f_{+}(u, v)-f_{-}(u)\right]+\nabla^{2} u, \\
& \frac{\partial v}{\partial t}=-g_{+}(u) v+g_{-}(u)(1-v) .
\end{aligned}
$$

Here $u$ and $v$ denote the extracellular cAMP concentration and the fraction of active receptors per cell, respectively. For computational simplicity, we take slightly simpler algebraic expressions for the kinetic terms than those of the original papers [2,8], which nevertheless retain the important biochemical characteristics of the latter. They read:

$$
\begin{array}{ll}
f_{+}(u, v)=\left(b v+v^{2}\right) \frac{a+u^{2}}{1+u^{2}} & \begin{array}{l}
\text { (autocatalytic cAMP production, mediated } \\
\text { by cAMP binding to active receptor), } \\
\text { (cAMP degradation), }
\end{array} \\
f_{-}(u)=d u & \text { (receptor desensitization rate per cell), } \\
g_{+}(u)=k_{+} u & \text { (receptor resensitization rate per cell), } \\
g_{-}(u)=k_{-} & \text {. }
\end{array}
$$

This system has the form of the standard equations describing an excitable medium [11], with $u$ taking the role of the propagator species and $v$ being the controller variable (Fig. 2). Wave solutions in one and two space dimensions have been computed which are in good quantitative agreement with the experimental observations [2].

System (1),(2) is a model for cAMP waves in a homogeneous layer of stationary cells. In situ, cells are motile, and the dynamics of the cell distribution is coupled to the chemical dynamics via chemotaxis in the cAMP gradient. The local cAMP production and degradation rates, in turn, will depend on the local cell density. Since no cell proliferation and appreciable cell death occur during aggregation, the conservation equation for the cell density $n$ simply takes the form

$$
\frac{\partial n}{\partial t}+\nabla \cdot J=0
$$

where $J$ denotes the cell flux. The standard chemotactic flux expression is

$$
J=-\mu \nabla n+\chi n \nabla u,
$$

accounting for random cell motility and chemotactic drift $[12,13]$. The parameters $\mu$ and $\chi$ denote the cell diffusion coefficient and the chemotactic coefficient, respectively. The form of the chemotaxis term is 
consistent with experiments which demonstrate that cells are able to orient and move persistently in stationary cAMP gradients [14]. The translation of the extracellular cAMP gradient into directed cell movement is rapid (characteristic time of a few seconds [15]), and therefore an instantaneous response to the gradient, $J \propto \nabla u$, is a reasonable approximation. In situ, however, cells move only in the fronts of the approximately symmetric cAMP pulses, and remain stationary in the wavebacks $[1,16]$. As it stands, relation (4) would predict cell movement both in wavefront and wavebacks. This would lead to the paradoxical conclusion that cells would show a small net translocation opposite to the direction of wave propagation and hence would not aggregate in the centre of the wave pattern. This so-called "chemotactic wave paradox" [16] implies that the chemotactic response of a cell cannot solely be determined by the local cAMP gradient. It is well-documented that the chemotactic machinery of a cell, too, exhibits desensitization upon exposure to cAMP. As the characteristic time scale of this adaptation reaction is roughly of the order of $1 \mathrm{~min}$ (with resensitization being somewhat slower than desensitization) [17], and thus of the order of the characteristic time for changes in cAMP concentration (cAMP wave speed $300 \mu \mathrm{m} \mathrm{min}{ }^{-1}$, halfwidth of a pulse $700 \mu \mathrm{m}$ ), we must include the time dynamics of the adaptation response in a mechanistic model. A reasonable first approximation is to associate the sensitivity of the chemotactic pathway towards the signal $\nabla u$, measured by the "transport" coefficient $\chi$, with the fraction of active receptors as a major desensitizing component of the chemotactic pathway [17], generally assuming a response threshold $A$ :

$$
\chi(v)=\chi 0 \frac{v^{m}}{A^{m}+v^{m}}, \quad m>1 .
$$

The dynamics of the cell distribution are then governed by the equation

$$
\frac{\partial n}{\partial t}=\nabla \cdot\left(\mu(n) \nabla n-\chi_{0} \frac{v^{m}}{A^{m}+v^{m}} n \nabla u\right) .
$$

We can account for the effect of cell-cell adhesion at higher densities [18] by a random motility coefficient of the form

$$
\mu(n)=\mu_{1}+\mu_{2} \frac{M^{r}}{M^{r}+n^{r}}, \quad r>1 .
$$

The parameter $M$ can be viewed as a threshold density for cell-cell adhesive effects. This form of the cell diffusion coefficient was used in the model in [9]. However, it turns out to be not essential for the patterning dynamics, and for the main numerical results we take $\mu=$ constant.

Höfer et al. [7] have shown that the chemotaxis-adaptation model (2) and (5) equips amoebae with a "short-term memory" for experienced cAMP concentrations: cells move in wavefronts but remain stationary in wavebacks. To account for receptor convection by the moving cells in (2), an additional term $-(J / n) \nabla v$ must be included in (2), cf. [7]. This convection term turns out to be very small for the situation in situ, as the cAMP wave speed is much greater than the cell velocity, and therefore we neglect it here.

Cell density will feed back into the cAMP kinetics. This is obvious for cAMP production, and is also the case for the degradation term, since cells carry and continuously secrete cAMP-hydrolyzing enzymes. We model these effects by including cell density factors in (1):

$$
\frac{\partial u}{\partial t}=\sigma\left[\phi(n) f_{+}(u, v)-\psi(n) f_{-}(u)\right]+\nabla^{2} u
$$

where $\phi(n)$ and $\Psi(n)$ are increasing functions of $n$. Specifically we take

$$
\phi(n)=\frac{n}{1-\kappa_{\phi} n /(K+n)},
$$




$$
\psi(n)=d_{1}+d_{2} \frac{n}{1-\kappa_{\psi} n /(K+n)} .
$$

These relationships are motivated as follows. In general, the rates of local cAMP production and degradation are proportional to $n /\left(1-\kappa n V_{c}\right)$, where $V_{c}$ and $1-\kappa$ denote the average volume occupied by a cell and the average fraction of residual extracellular volume in a confluent cell layer, respectively [3,9]. However, this would require a three-dimensional formulation of the problem when cells start to pile at the aggregation centre. To circumvent this difficulty within our two-dimensional framework, we let the denominator term, accounting for volume exclusion by the cells, approach a constant value when the cell density, measured in number of cells per area, becomes large. A small background activity of secreted extracellular phosphodiesterase independent of cell density is added to the degradation term.

For the majority of parameter values numerical estimates can be extracted from the experimental literature. For the cAMP signalling system this has been done by Martiel and Goldbeter [8], and recently for a very detailed model by Tang and Othmer [10]. For the crucial cell motility parameters, $\chi$ and $\mu$, measurements exist for leukocytes, another amoeboid cell type, which give an indication of the typical range of these parameter values [19-21]. To get an estimate for Dictyostelium, the chemotactic coefficient has to be weighted with a characteristic chemoattractant concentration (which differ by an order of magnitude for Dictyostelium and leukocytes). Dictyostelium shows a strong chemotactic response, and a value for $\chi_{\text {dim }}$ somewhat higher than the maximum obtained for leukocytes yields a cell velocity profile in the cAMP wave very close to that observed experimentally [1]; cf. Fig. 3d below. Key dimensional parameters are listed in Table 1. The nondimensionalised form in which the model equations are given is defined in Table 2. Note that, taking the length scale to be a typical diffusion length of cAMP, $\chi_{0}$ is roughly of order 1 whereas $\mu(n)$ is of order $10^{-2}$, that is, (5) can be viewed as an advection equation with a small diffusive smoothing term. The "threshold parameters" $K$ and $A$ in relative units of the initial cell density and in terms of $R_{T}$, respectively, are chosen to give good comparison of the numerical simulations with in situ observations (Section 4).
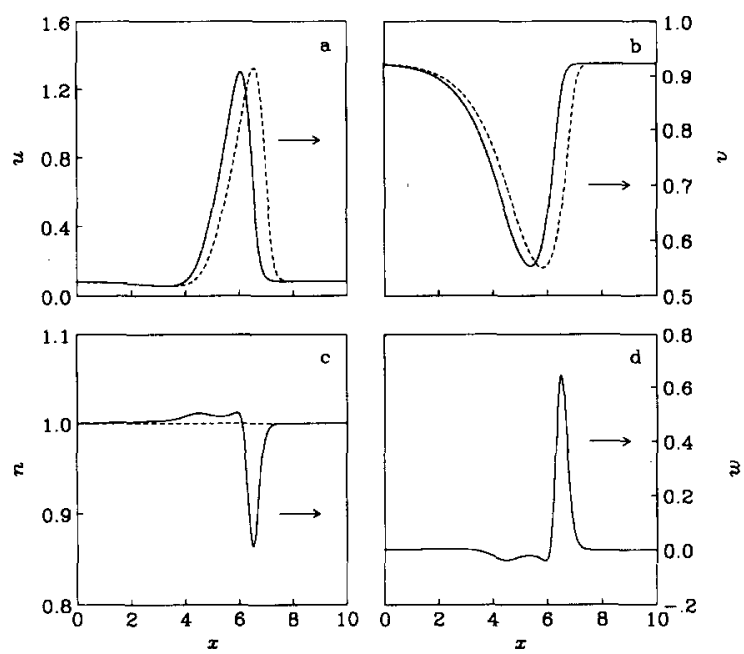

Fig. 3. Travelling pulse solution of system (2),(5),(6) in one dimension (solid line), compared to wave solution of (2),(6) with clamped cell density, $n=1$ (dashed line); (a) cAMP concentration, (b) fraction of active receptors, (c) cell density, and (d) cell velocity. Parameter values as in Table 2. The numerically determined wavespeed for the pulse in the full system is 4.3 , which is marginally slower (ca. $4 \%$ ) than the pulse at clamped cell density (profiles are shown at $t=3.0$ after starting from the same point). The $u$ and $v$-profiles in both cases are practically identical. 
Table 1

Dimensional values of key parameters

\begin{tabular}{|c|c|c|c|}
\hline Key dimensional parameter & Experimental range & $\begin{array}{l}\text { Value used in } \\
\text { calculations }\end{array}$ & Reference \\
\hline overall rate of adenylate & & & [8], \\
\hline $\begin{array}{l}\text { cyclase pathway per unit } \\
\text { cell density, } k_{a}\end{array}$ & $5 \times 10^{-7}-10^{-3} \mathrm{Mmin}^{-1}$ & $1.4 \times 10^{-5} \mathrm{M} \mathrm{min}^{-1}$ & $\frac{\sigma q k_{1}}{K_{R} h\left(k_{i}+k_{t}\right)}$ \\
\hline $\begin{array}{l}\text { rate of phosphodiesterase } \\
\text { per unit cell density, } k_{p}\end{array}$ & $2.5-12.5 \mathrm{~min}^{-1}$ & $7.3 \min ^{-1}$ & [8], ke \\
\hline desensitization rate, $l_{+}$ & $0.22 \mu \mathrm{M}^{-1} \min ^{-1}$ & $2.0 \mu \mathrm{M}^{-1} \min ^{-1}$ & [8] \\
\hline resensitization rate, $l_{-}$ & $0.104 \mathrm{~min}^{-1}$ & $1.0 \mathrm{~min}^{-1} \mathrm{a}$ & {$[8]$} \\
\hline $\begin{array}{l}\text { dissociation constant of } \\
\text { cAMP-active receptor } \\
\text { complex, } K_{R}\end{array}$ & $10^{-7} \mathrm{M}$ & $10^{-7} \mathrm{M}$ & {$[8], K_{R}$} \\
\hline no. of receptors per cell, $R_{T}$ & $5 \times 10^{4}$ & $5 \times 10^{4}$ & {$[17]$} \\
\hline diffusivity of cAMP, $D$ & $4 \times 10^{-6} \mathrm{~cm}^{2} \mathrm{~s}^{-1}$ & $3 \times 10^{-6} \mathrm{~cm}^{2} \mathrm{~s}^{-1 \mathrm{~b}}$ & {$[35]$} \\
\hline $\begin{array}{l}\text { chemotactic coefficient } \times \\
\text { typical chemoattractant } \\
\text { concentration, } \chi_{\mathrm{dim}} u_{\mathrm{char}} \mathrm{c}\end{array}$ & $10^{-7}-10^{-6} \mathrm{~cm}^{2} \mathrm{~s}^{-1}$ & $\begin{array}{l}1.5 \times 10^{-6} \mathrm{~cm}^{2} \mathrm{~s}^{-1}= \\
3 \mathrm{~cm}^{2} \mathrm{M}^{-1} \mathrm{~s}^{-1}\left(5 K_{R}\right)\end{array}$ & $|19,20|$ \\
\hline random cell migration, $\mu_{\mathrm{dim}}$ & $10^{-8}-10^{-7} \mathrm{~cm}^{2} \mathrm{~s}^{-1}$ & $3.6 \times 10^{-8} \mathrm{~cm}^{2} \mathrm{~s}^{-1}$ & {$[19]$} \\
\hline
\end{tabular}

a The estimates for these parameters are obtained by multiplying the relevant rate constants in [8], $k_{2}$ and $k_{1}$, by a characteristic cAMP concentration in a wave $\left(10^{-6} \mathrm{M}\right)$. The values used in our calculations are the ones used by Tyson et al. [2].

${ }^{b}$ We take $75 \%$ of the value in water, accounting for higher viscosity of the medium on agar.

c Experimental values given are for human leukocytes; for comparison, the values for the chemotactic coefficient are multiplied with the corresponding chemoattractant concentrations.

Table 2

Parameters of the nondimensionalised model

\begin{tabular}{llc}
\hline Name & Definition & Numerical value \\
\hline time scale, $\tau$ & - & $2.5 \mathrm{~min}$ \\
space scale & $(D \tau)^{1 / 2}$ & $212 \mu \mathrm{m}$ \\
cAMP scale & $5 K_{R}$ & $0.5 \mu \mathrm{M}$ \\
active receptor scale & $R_{T}$ & $5 \times 10^{4}$ \\
$\sigma$ & $k_{a} \tau /\left(5 K_{R}\right)$ & 70.0 \\
$a$ & $a$ & 0.014 \\
$b$ & $\mathrm{a}$ & 0.2 \\
$d_{1}$ & $0.1 \tau k_{p} / \sigma$ & 0.026 \\
$d_{2}$ & $0.9 \tau k_{p} / \sigma$ & 0.234 \\
$k_{+}$ & $5 K_{R} \tau l_{+}$ & 2.5 \\
$k_{-}$ & $\tau l-$ & 2.5 \\
$\chi_{0}$ & $\chi \operatorname{dim}\left(5 K_{R}\right) / D$ & 0.5 \\
$\mu$ & $\mu_{\mathrm{dim}} / D$ & 0.012 \\
$A$ & - & 0.72 \\
$K$ & - & 0.8 \\
$\kappa_{\phi}, \kappa_{\psi}$ & - & 0.7 \\
$m$ & - & 10.0 \\
\hline
\end{tabular}

${ }^{a}$ Numerical values chosen to obtain a kinetic response of the simplified adenylate cyclase pathway kinetics similar to the kinetics derived from a specific reaction scheme in [8]. 


\section{Chemotactic instability of wave trains}

System (2),(6) with $n=$ constant is identical to (1),(2), which has the properties of a classical excitable medium [11], and in particular exhibits periodic plane wave solutions [2]. We now look for periodic wave solutions to the full system $(2),(5),(6)$. They are governed by

$$
\begin{aligned}
& c\left(N-n_{0}\right)+\mu(N) \frac{d N}{d z}-\chi(V) N \frac{d U}{d z}=0, \\
& \frac{d^{2} U}{d z^{2}}+c \frac{d U}{d z}+\sigma f(N, U, V)=0, \\
& c \frac{d V}{d z}+g(U, V)=0
\end{aligned}
$$

where

$$
\begin{aligned}
& f(N, U, V)=\phi(N) f_{+}(U, V)-\psi(N) f_{-}(U), \quad g(U, V)=k_{+} U V-k_{-}(1-V), \\
& \left.\begin{array}{l}
n(x, y, t)=N(z)=N(z+\tilde{\Lambda}) \\
u(x, y, t)=U(z)=U(z+\tilde{\Lambda}) \\
v(x, y, t)=V(z)=V(z+\tilde{\Lambda})
\end{array}\right\} z=x-c t,
\end{aligned}
$$

and $c$ and $\tilde{\Lambda}$ denote wave speed and wavelength, respectively. As $\mu \ll \chi_{0}$ (cf. Table 2), we obtain from (7) the approximation

$$
N(z)=\frac{n_{0} c}{c-w(z)}
$$

where $w(z)=\chi(V(z)) d U(z) / d z$ is the average chemotactic cell velocity. Here the meaning of $n_{0}$ in (7) as the unperturbed homogeneous cell density becomes apparent. As we know from experiment that $w(z) / c \leq 0.1$, the homogeneous cell density is perturbed in the wavefront by about $10 \%$. Thus we can expect travelling wave solutions of $(2),(5),(6)$ close to those of (1),(2), which is confirmed by numerical simulations (Fig. 3). Relation (10) is consistent with experimental observations. Alcantara and Monk report that they could not observe systematic differences in cell density in the layer of amoebae during the initial stages of aggregation [5]. The amoebae were practically homogeneously distributed, and the movement response in the cAMP waves did not result in noticeable changes in cell density.

In line with previous analytical and numerical results $[2,11]$, our numerical simulations indicate that largeamplitude wave propagation in one space dimension is a stable phenomenon. In particular, random initial perturbations around the homogeneous cell density of about \pm 0.1 perturb the wave profile only very slightly and decay with time. However, in situ waves propagate over a plane surface, and this situation may change. The two-dimensional plane and spiral wave solutions of the cAMP signalling system (1),(2), calculated by Tyson et al. [2], again appear to be stable. This is in good agreement with the experimental situation at the onset of aggregation, during which both stable target patterns and spirals are observed.

To investigate the stability of wave propagation in the full model $(2),(5),(6)$ in two space dimensions, we add a small perturbation to the solutions of (7)-(9),

$$
\begin{aligned}
& n(x, y, t)=N(z)+\hat{n}(z, y), \\
& u(x, y, t)=U(z)+\hat{u}(z, y), \\
& v(x, y, t)=V(z)+\hat{v}(z, y) .
\end{aligned}
$$


Here we assume a special form of the perturbation which has only implicit time dependence via the travelling wave coordinate $z$. We will see that the analysis of this special kind of perturbation provides us with an interesting insight into the nature of the patterning mechanism. The reasoning behind this is as follows. As wave propagation in the chemical subsystem (1),(2) is stable, that is, perturbations of the concentration profile are rapidly damped rather than propagated with the wave and amplified (the typical phenomenology of wavefront instability in chemical systems [22]), a possible instability must be linked to the dynamics of cell movement. These are forced by the cAMP waves, which may cause inhomogeneities in cell density, as the main source of stochasticity in the system, to grow. These inhomogeneities will not propagate with the waves, however, as $w \ll c$. Therefore we can expect a perturbation to evolve essentially in the travelling-wave frame. Subsequent numerical calculations confirm this picture [Section 4, Fig. 7d]. From $(2),(5),(6)$ we obtain the following linear system governing the evolution of the small perturbation $(\hat{n}, \hat{u}, \hat{v})$ :

$$
\begin{aligned}
& \frac{\partial}{\partial z}\left[c \hat{n}+\mu(N) \frac{\partial \hat{n}}{\partial z}+\mu_{n}(N) \hat{n} \frac{\partial N}{\partial z}-\chi(V) N \frac{\partial \hat{u}}{\partial z}-\left[\chi(V) \hat{n}+\chi_{v}(V) \hat{v} N\right] \frac{\partial U}{\partial z}\right] \\
& \quad+\mu(N) \frac{\partial^{2} \hat{n}}{\partial y^{2}}-\chi(V) N \frac{\partial^{2} \hat{u}}{\partial y^{2}}=0, \\
& \frac{\partial^{2} \hat{u}}{\partial z^{2}}+c \frac{\partial \hat{u}}{\partial z}+\frac{\partial^{2} \hat{u}}{\partial y^{2}}+\sigma\left[f_{n}(N, U, V) \hat{n}+f_{u}(N, U, V) \hat{u}+f_{v}(N, U, V) \hat{v}\right]=0, \\
& c \frac{\partial \hat{v}}{\partial z}-k_{+} V \hat{u}-\left(k_{+} U+k_{-}\right) \hat{v}=0 .
\end{aligned}
$$

Indices on the functions $\mu, \chi$, and $f$ denote partial derivatives with respect to the index variable. Separable solutions to (11)-(13) can be sought in the form $a(z) \exp \{i q y\}$, that is, Fourier modes in the $y$-direction (i.e. parallel to the wavefronts) with a spatial wavenumber $q$ and an amplitude $a$ evolving in the travelling wave coordinate. This yields the following ordinary differential equation system for $a=\left(a_{(n)}, a_{(u)}, a_{(v)}\right)^{\top}$ :

$$
\frac{1}{c^{2}}\left(\begin{array}{c}
\eta^{\prime \prime}(\xi) \\
a_{(u)}^{\prime \prime}(\xi) \\
0
\end{array}\right)-\boldsymbol{a}^{\prime}(\xi)+\left(\begin{array}{ccc}
-\mu(N) q^{2} \chi(V) N q^{2} & 0 \\
\sigma f_{n} & \sigma f_{u}-q^{2} & \sigma f_{v} \\
0 & -k_{+} V & -\left(k_{+} U+k_{-}\right)
\end{array}\right) \boldsymbol{a}(\xi)=0
$$

where we have rescaled $z=-c \xi$ and used the abbreviation

$$
\eta^{\prime \prime}=\left[\mu(N) a_{(n)}^{\prime}+\mu_{n}(N) a_{(n)} N^{\prime}-\chi(V) N a_{(u)}^{\prime}-\left[\chi(V) a_{(n)}+\chi_{v}(V) a_{(v)} N\right] U^{\prime}\right]^{\prime} .
$$

Here ' denotes differentiation with respect to $\xi$. Eq. (14) is a linear non-autonomous system; the $\xi$-dependent coefficients are $\Lambda=\tilde{\Lambda} / c$-periodic functions via $N(\xi), U(\xi)$ and $V(\xi)$. It can be interpreted as looking at a strip at $x=x_{0}$ parallel to the wavefronts, and following the evolution of an initial perturbation in this strip, as the periodic waves pass through it. Thus our analysis will capture patterning perpendicular to the direction of wave propagation, without at present addressing the more involved issue of pattern selection on the two-dimensional domain. Also note that $1 / c^{2} \approx 0.05 \ll 1$ (cf. Fig. 3), and hence the corresponding term will be negligible.

Let $\Theta(\xi)$ denote a fundamental matrix of (14). Then standard theory gives the following stability criterion: The general solution of (14) decays to (grows away from) the trivial solution, if the linear mapping

$$
\boldsymbol{\Theta}\left(\xi_{0}\right) \stackrel{M}{\rightarrow} \Theta\left(\xi_{0}+\Lambda\right)
$$

is contracting (expanding in at least one direction). This can be established straightforwardly from the eigenvalues of a particular realization of the matrix $\boldsymbol{M}$, which are independent of the choice of $\xi_{0} . \boldsymbol{M}$ is called the monodromy matrix, and its eigenvalues $\rho_{i}$ are the Floquet multipliers of the system. 


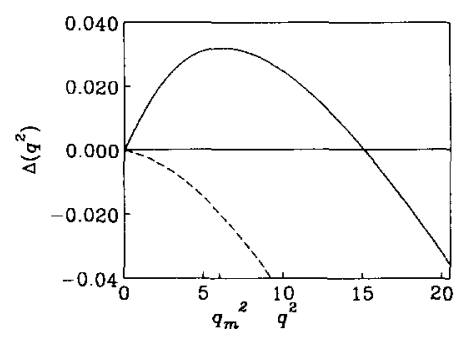

Fig. 4. Numerically determined dispersion relation (15); Parameter set as in Table 2, and $\Lambda=2.9$ (solid line). A range of wavenumbers is unstable, with a maximum of the dispersion curve at $q_{m}=2.5$. (The neutral mode at $q=0$ is the translational mode.) Contrast this with the stable situation (dashed line), obtained with the same parameter values, except $\chi_{0}=0.2$ (weaker chemotactic response).

We can calculate the $\rho_{i}$ and the corresponding eigenvectors numerically by computing a travelling wave solution $(N(z), U(z), V(z))$ from (2),(5),(6), with a period $\Lambda$ in the range of observed periods, and subsequently solving (14) for a complete set of linearly independent initial conditions over one period. This yields a particular representation of $\boldsymbol{M}$, from which eigenvalues and eigenvectors are readily obtained. We repeat this procedure for a range of $q$-values (holding all other parameters at the values given in Table 1), and so obtain the dispersion relation for patterning in the $y$-direction,

$$
\Delta\left(q^{2}\right)=\frac{1}{\Lambda} \ln \rho_{1}\left(q^{2}\right)
$$

where $\rho_{1}$ denotes the largest Floquet multiplier (neglecting the $1 / c^{2}$-term which contributes two positive eigenvalues irrespective of the forcing by the waves); it is customary to give the numerical value of the Floquet exponent, $\ln \rho$, normalized by the forcing period. The result is depicted in Fig. 4. We find that for a large range of wavenumbers the leading Floquet exponent is real and positive, corresponding to a Floquet multiplier $\rho_{1}>1$. Thus the initially uniform situation (for which the travelling waves at fixed cell density have been computed $[2,3])$ is unstable from the outset. The maximum of the dispersion relation occurs at a wavenumber $q_{m}$ of about 2.5 , which corresponds to a wavelength of approximately $2.5(530 \mu \mathrm{m})$. Hence this analysis suggests that the coupled dynamics of cAMP wave propagation and cell movement exhibit a patterning instability perpendicular to the direction of wave propagation, whose intrinsic wavelength (assuming that the fastest growing linear mode dominates the pattern) is roughly an order of magnitude larger than a typical cell diameter (ca. 10-20 $\mu \mathrm{m})$. The components of the eigenvector to $\rho_{1}\left(q_{m}\right)$ corresponding to the amplitudes of the perturbations in $n, u$ and $v$ have the approximate ratio 1:0.01:0.004. Therefore the instability will largely appear as the growth of a pattern in cell density. Thus this linear stability analysis gives a clear indication that our relatively simple model can capture the mechanism of cell stream formation.

\section{An analytic caricature}

Eq. (14) can only be treated numerically, and so it is difficult to establish the role that different terms play in either stabilizing or destabilizing the plane waves. Thus we derive a heuristic caricature of the model which permits analytic treatment. In line with the above stability analysis, we begin by writing the model equations $(2),(5),(6)$ in $(\xi, y)$-coordinates, yielding the (rescaled) nonlinear correlate to (11)-(13). We neglect terms $\propto 1 / c^{2} \ll 1$, and write in analogy to the resulting system the following caricature equations

$$
\frac{\partial n(\xi, y)}{\partial \xi}=\frac{\partial}{\partial y}\left(\mu \frac{\partial n}{\partial y}-\chi(\xi) n \frac{\partial u}{\partial y}\right)
$$




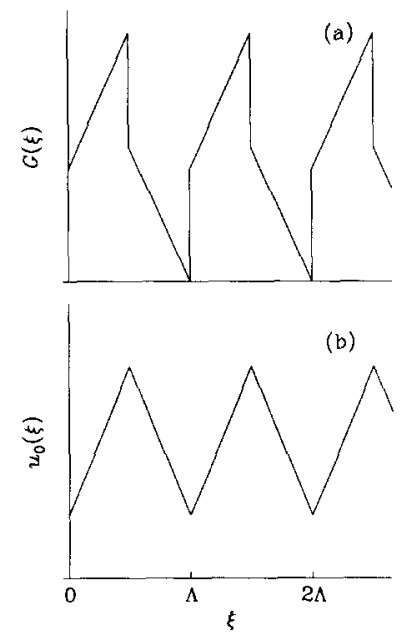

Fig. 5. (a) Piecewise linear cAMP production rate $G(\xi)$, and (b) $y$-independent solution $u_{0}(\xi)$ of the caricature (16)-(19).

$$
\frac{\partial u(\xi, y)}{\partial \xi}=n[G(\xi)-d u]+\delta \frac{\partial^{2} u}{\partial y^{2}} .
$$

The $v$-dynamics have been replaced by the $\Lambda$-periodic positive forcing terms $G(\xi)$ for the cAMP production kinetics and $\chi(\xi)$ for chemotactic adaptation. Both production and degradation rates are taken to be proportional to the cell density. In order that all the relevant parameters appear in the final result, dimensional length has been rescaled with an arbitrary constant, and $\delta$ denotes the nondimensionalised diffusivity of cAMP. We will see below that for the specific choice of the following piecewise linear cAMP production rate the stability analysis of (16), (17) can be carried out explicitly:

$$
G(\xi)=p\left\{\begin{array}{cc}
2+d n_{0}(\xi-j \Lambda) & \text { if } \xi \in I_{j}^{+} \\
d n_{0}((j+1) \Lambda-\xi) & \text { if } \xi \in I_{j}^{-}
\end{array},\right.
$$

where $I_{j}^{+}=(j \Lambda,(j+1 / 2) \Lambda)-$ wavefront, and $I_{j}^{-}=((j+1 / 2) \Lambda,(j+1) \Lambda)-$ waveback, $j=0,1,2, \ldots ;$ the parameter $p$ essentially determines the forcing amplitude, cf. Fig. 5. Chemotactic adaptation in the waveback is included by setting

$$
\chi(\xi)=\left\{\begin{array}{ll}
\bar{\chi} & \text { if } \xi \in I_{j}^{+} \\
0 & \text { if } \xi \in I_{j}^{-}
\end{array} .\right.
$$

System (16)-(19) accounts in a crude way for the essential features of the full model, namely, periodic chemoattractant production and adapting chemotaxis (in the travelling wave frame $\xi=t-x / c$ ). It has the $y$-independent (homogeneous) solution $n=n_{0}$, and

$$
u_{0}(\xi)=\left\{\begin{array}{cl}
p\left[1 / d+n_{0}(\xi-j \Lambda)\right] & \text { if } \xi \in I_{j}^{+} \\
p\left[1 / d+n_{0}(\xi-(j+1) \Lambda)\right] & \text { if } \xi \in I_{j}^{-} .
\end{array},\right.
$$

that is, periodic "zig-zag" cAMP wave trains on a homogeneous cell layer which mimic the observed cAMP waves (Fig. 5). Clearly, this solution is stable to small perturbations in the $y$-direction in the absence of chemotaxis. When chemotaxis is present, the time evolution of a small disturbance $\propto \exp \{i q y\}$ is governed by the linear system

$$
\boldsymbol{b}^{\prime}=\left(\begin{array}{cc}
-\mu q^{2} & \chi(\xi) n_{0} q^{2} \\
F(\xi) & -d n_{0}-\delta q^{2}
\end{array}\right) \boldsymbol{b}
$$




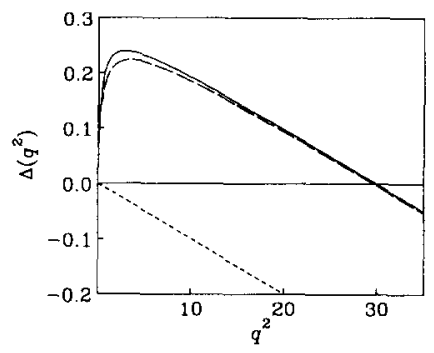

Fig. 6. Dispersion relation of the caricature (16)-(19) in the stable $(p \bar{\chi}=0.001, A=0.1$, small dashes) and in the unstable $(p \bar{\chi}=0.6$, $\Lambda=0.0001$, solid line, and $p \bar{X}=0.6, A=20.0$, long dashes) regime; other parameters: $\mu=0.01, \delta=1.0, d=0.1$ and $n_{0}=1.0$. For the unstable case, (22) predicts a zero of the dispersion curve at $q^{2}=29.9$, which is accurate also for large cAMP wavelength.

which is the caricature analogue to $(14) . F(\xi)=G(\xi)-d u_{0}(\xi)$ denotes the piecewise constant function $\pm p$, for $\xi=I_{j}^{ \pm}$. Thus the Floquet analysis of (21), that is, the construction of the caricature dispersion relation, can be carried out explicitly; this is done in the Appendix. We find that in the unstable regime the dispersion relation has the same simple shape as the dispersion relation computed numerically for the full system (Fig. 6). In the limiting cases $\Lambda \rightarrow 0$ and $\Lambda \rightarrow \infty$, simple stability conditions can be derived. In the case $A \rightarrow 0$ the trivial solution of (21) is unstable to a perturbation $\propto \exp \{i q y\}, q \neq 0$, if

$$
p \bar{\chi} n_{0}>2 \mu\left(d n_{0}+\delta q^{2}\right)
$$

and stable when the inequality is reversed. The criterion (A.10) for $\Lambda \rightarrow \infty$ practically coincides with (22) when, as it is the case for our system, $\mu \ll \delta$. This suggests that the instability criterion (22) holds approximately also for $0<A<\infty$, for which an exact, but rather involved instability condition, (A.8) with (A.5)-(A.7), is obtained. This is confirmed by sample calculations with specific parameters. It clearly shows that the dynamics parallel to the wavefronts are determined by the competition between aggregative tendencies (chemotaxis and cAMP production) and dispersive tendencies (cAMP and cell diffusion, cAMP degradation); the homogeneous situation becomes unstable when the former dominate.

Interestingly, condition (22) is almost identical to the criterion for chemotaxis-driven instability for the standard non-oscillatory system introduced by Keller and Segel [12]. In our notation, the linearization of the Keller-Segel system is (21) with $F(\xi)=p=$ constant, $\chi(\xi)=\bar{\chi}=$ constant and $d n_{0}$ replaced by $d$ (the degradation rate in the non-oscillatory case is assumed independent of the cell density). The corresponding instability criterion reads $p \bar{\chi} n_{0}>\mu\left(d+\delta q^{2}\right)$, that is, despite the qualitatively different system dynamics in both cases the instability criteria suggest a very similar interpretation of the basic instability mechanisms. Note, however, an obvious difference: in the non-oscillatory case no instability is obtained, when, as in (17), chemoattractant production and degradation rates have identical cell density dependence. On the other hand, variation in cell density does not greatly influence the stability of the oscillatory system (16),(17).

\section{Numerical simulations}

To explore the consequences of the instability revealed by the linear analysis, we solve the full nonlinear model $(2),(5),(6)$ numerically on a rectangular domain. We use an alternating direction implicit scheme for the diffusion operator, coupled with an upwind scheme for the advection part [23]. Tests with different grid sizes and time steps showed that nondimensional space and time steps of 0.2 and 0.005 , respectively, yield accurate results both with respect to the spiral wave dynamics on a stationary cell layer and the patterning 
dynamics when cell movement is included. Conservation of total cell number was found to be guaranteed within a range of $\pm 1 \%$ for the simulations shown.

A typical result is shown in Fig. 7. A spiral wave develops from a disrupted wavefront. To approximate the effect of random fluctuations in cell distribution, initially a small random perturbation is added at each meshpoint to the homogeneous cell density. This perturbation is amplified on an intrinsic length scale, leading to the formation of alternating "stripes" of increased and decreased cell density along the radial direction from the aggregation centre. This process is apparent in the sequence of snapshots from 5 to $35 \mathrm{~min}$ in Fig. 7, and is analogous to the in situ observations [transition from Figs. 1a-d]. The wavelength of the pattern is about 2.4 [Fig. 7c], and is thus in good agreement with the theoretical prediction of 2.5 for the fastest growing linear mode (Section 3). Subsequently the stripes of increased cell density develop into the cell streams observed experimentally (Figs. 7a, $75 \mathrm{~min}$, and le,f respectively]. Consistent with the theoretical analysis and experiment, pattern appears first in the centre and grows outwards with each wave.

The growing cell streams in turn alter the propagation conditions for the cAMP waves. An increase in cell density is initially equivalent to an increase in $\sigma$ and hence locally increased excitability of the medium. This leads to two interesting phenomena in the model, both of which are in fact observed in the natural system. They concern systematic changes of the "global" properties of the cAMP spiral, such as wavelength and wave speed, and the structure of the core region of the spiral.

As cells aggregate in the centre and cell streams start to develop, the excitability of the medium is increased in the centre of the pattern. In particular, this leads to a gradual increase in the rotation frequency of the spiral core. At any given point (outside the core) this appears as an increased frequency of wave emission from the centre of the pattern. Cell density and hence excitability are relatively unchanged outside the central region of the pattern where the cell layer just starts to break up into streams. Thus the propagation speeds gets "pushed" downwards on the dispersion curve [11], and successive waves slow down gradually (with each wave having a practically constant velocity after leaving the central region, where curvature effects also influence the wave speed), while the wavelength of the spiral pattern decreases, cf. Fig. 8. This slowing down and decrease in wavelength is typical of Dictyostelium spiral patterns in situ [24]. On the other hand, such a phenomenon could not be observed in a homogeneous two-dimensional excitable medium, where usually a unique spiral geometry and rotation period [11] (or, for special non-monotonic dispersion relations, discrete alternative spirals [25]) exist. After this initial time interval, one actually observes intermittent propagation failure in the model, together with a small relocation of the spiral core away from the region of highest excitability, before a normal cAMP spiral is re-established immediately afterwards (though with an increasingly rugged concentration profile as a result of cell concentration along streams). For this time period, experimental data on cAMP profiles are not available for comparison, and as streams develop, the different light scattering responses of stationary and moving cells in monolayers of cells can no longer be used as indicators of wave position.

At low initial cell densities the numerical simulations predict the formation of a central hole which may be transient or continues to exist throughout aggregation [Fig. 9a]. Again this is not a model artefact; hole formation can also be observed in situ [Fig. 9b]. It appears to be linked to the movement of the spiral core, which in our model simulations (and also in the simulations by Tyson et al. [2] without cell movement) follows a circular path. Its diameter increases with decreased excitability, that is, with a decrease in $\sigma$ or in initial cell density. If the initial cell density is sufficiently low, the diameter of the core path is large enough for the core region to be depleted of cells by chemotaxis before cell aggregation limits core migration, with random cell movement not being able to counteract this process. A ring of cells is then formed, and the spiral core becomes locked into the path prescribed by this ring, despite overall increase in cell density in the centre with time. Alternatively, excitability can be lowered with caffeine treatment, which also induces central holes [1]. Caffeine partly inhibits the adenylate cyclase pathway and in the model would lead to an dereased value 

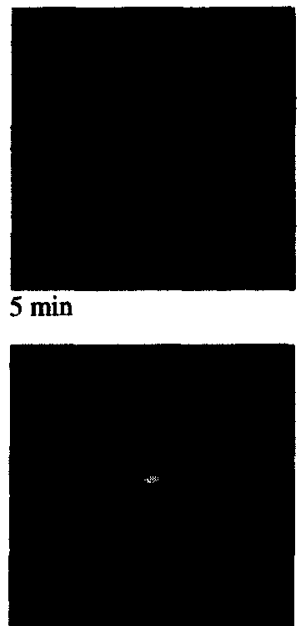

$25 \mathrm{~min}$

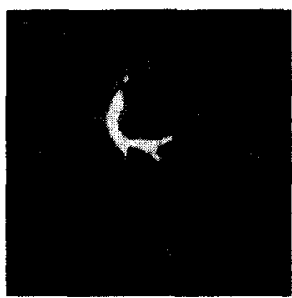

$55 \mathrm{~min}$

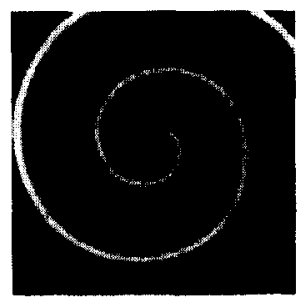

5 min

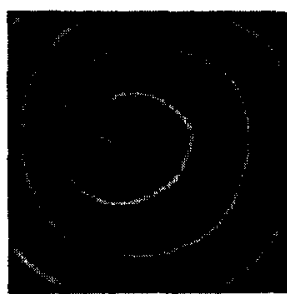

$25 \mathrm{~min}$

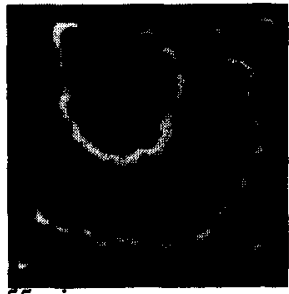

$55 \mathrm{~min}$

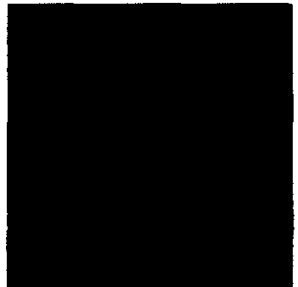

$10 \mathrm{~min}$

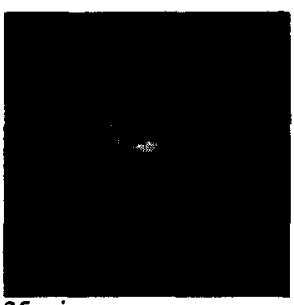

$35 \mathrm{~min}$

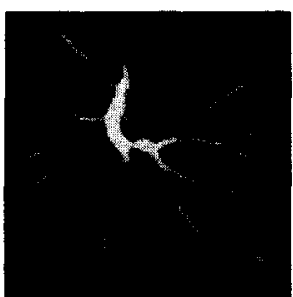

$65 \mathrm{~min}$
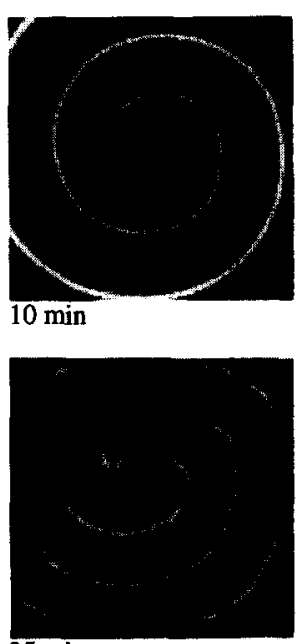

$35 \mathrm{~min}$

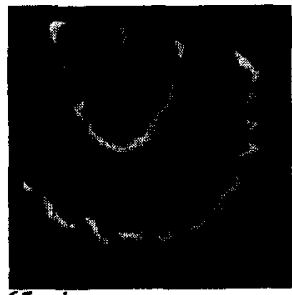

$65 \min$

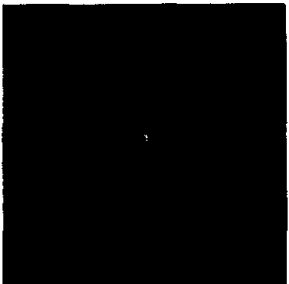

$15 \mathrm{~min}$

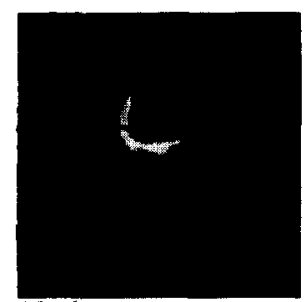

$45 \mathrm{~min}$

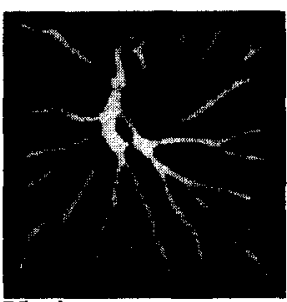

75 min

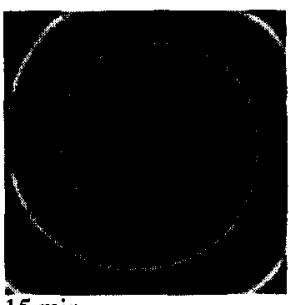

$15 \mathrm{~min}$

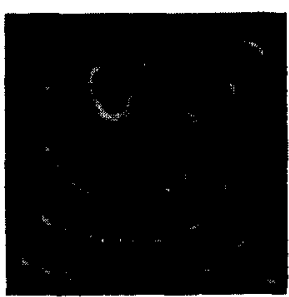

$45 \mathrm{~min}$
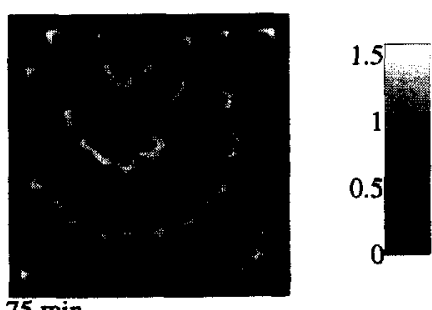

2.5

1.5

(b)

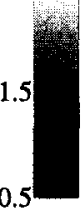

Fig. 7. 

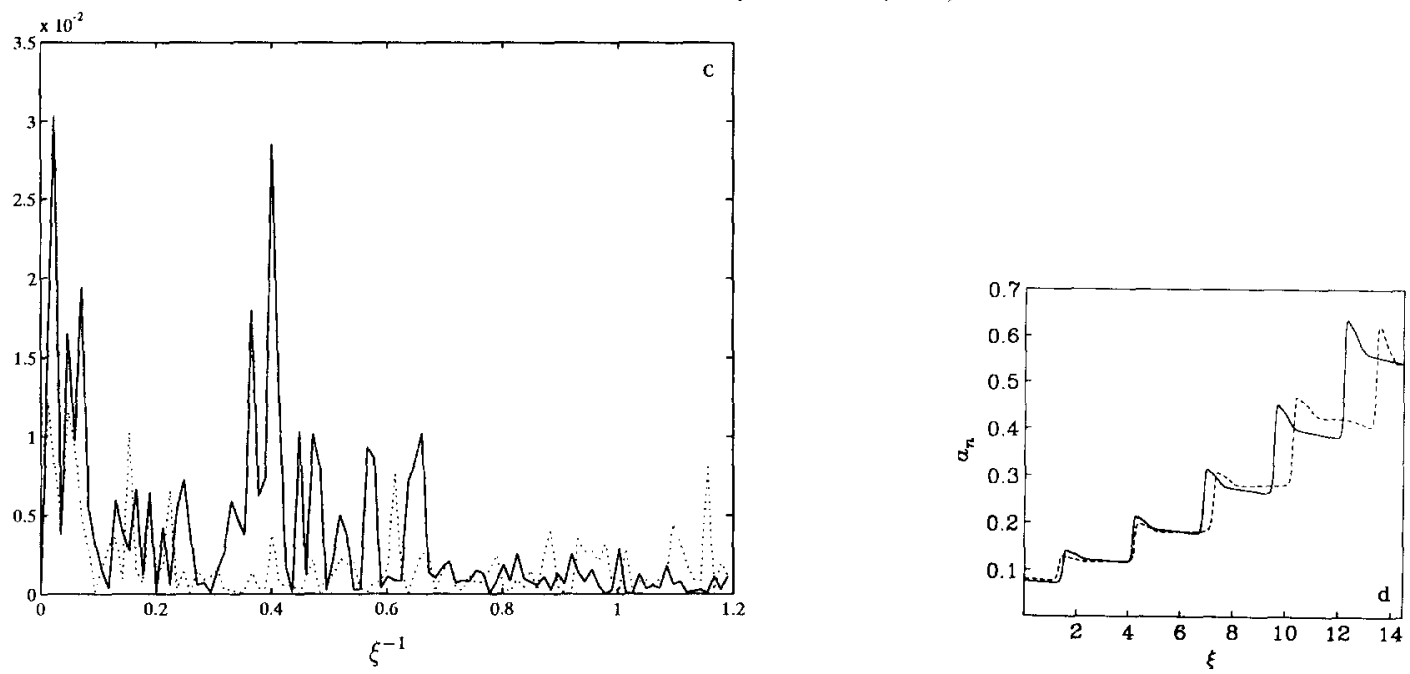

Fig. 7. Spatio-temporal evolution of (a) cell density, and (b) cAMP concentration in a numerical simulation of system (2),(5),(6). The dimensional domain size is $6.4 \mathrm{~mm} \times 6.4 \mathrm{~mm}(151 \times 151$ meshpoints $)$, and snapshots are taken at the times indicated. Initial conditions were chosen to be a plane wavefront with a free end at the centre of the domain and homogeneous cell density (1.0) with a random perturbation between -0.075 and 0.075 added at every mesh point. Boundary conditions are zero-flux. (c) Power spectrum of the cell density distribution on the curve defined by the waveback $(u=0.5)$ at $25 \mathrm{~min}$ (solid line), and of the initial cell density along the same curve (dotted line). One can clearly see the growth of a pattern with $q \approx 2.4$ (ca. $510 \mu \mathrm{m}$ ), in good agreement with the prediction of the linear stability analysis. (The maximum at small $\xi^{-1}$ corresponds to a full rotation along the sampling curve.) (d) Evolution of the amplitude of the cell density perturbation in a simulation with periodic plane waves plotted in the $\xi=-x+t / c$ coordinate; solid line: fixed $x, t$ varying; dashed line: fixed $t$ with $x$ varying across the domain. The numerical results clearly support our assertion that the perturbation essentially evolves in the travelling wave frame (differences at increasing $\xi$ are mainly due to slight variation in wave speed with time). Parameter values as in Table 2 .
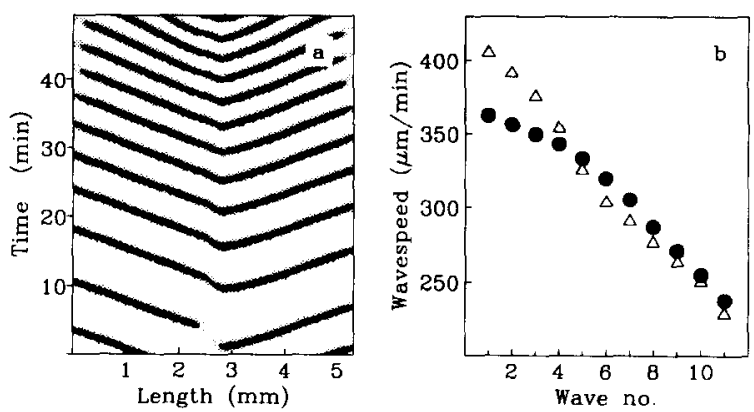

Fig. 8. (a) Evolution of cAMP concentration in a spatial slice through the aggregation territory; (b) corresponding wave velocities ( $\bullet$ ), compared to the experimental data in [24], Fig. $3(\triangle)$. The spatial slice is taken parallel to the horizontal boundary and through the wave core. In contrast, a spiral wave on a stationary cell layer with constant density 1.0 develops, after a short initial transient, to a stationary shape with wavespeed ca. $360 \mu \mathrm{m} / \mathrm{min}$ outside the core region. Parameter set as in Table 2, with $\kappa_{\phi}=0.6, \kappa_{\psi}=8.0$.

of $\sigma$, and again to the prediction of hole formation (for a more detailed discussion of the core dynamics see [9]).

\section{Discussion}

In 1970, Keller and Segel introduced the idea of the formation of cell aggregation patterns via a dynamic instability in a chemotaxis system [12]. Since then their chemotaxis equations have been applied successfully 

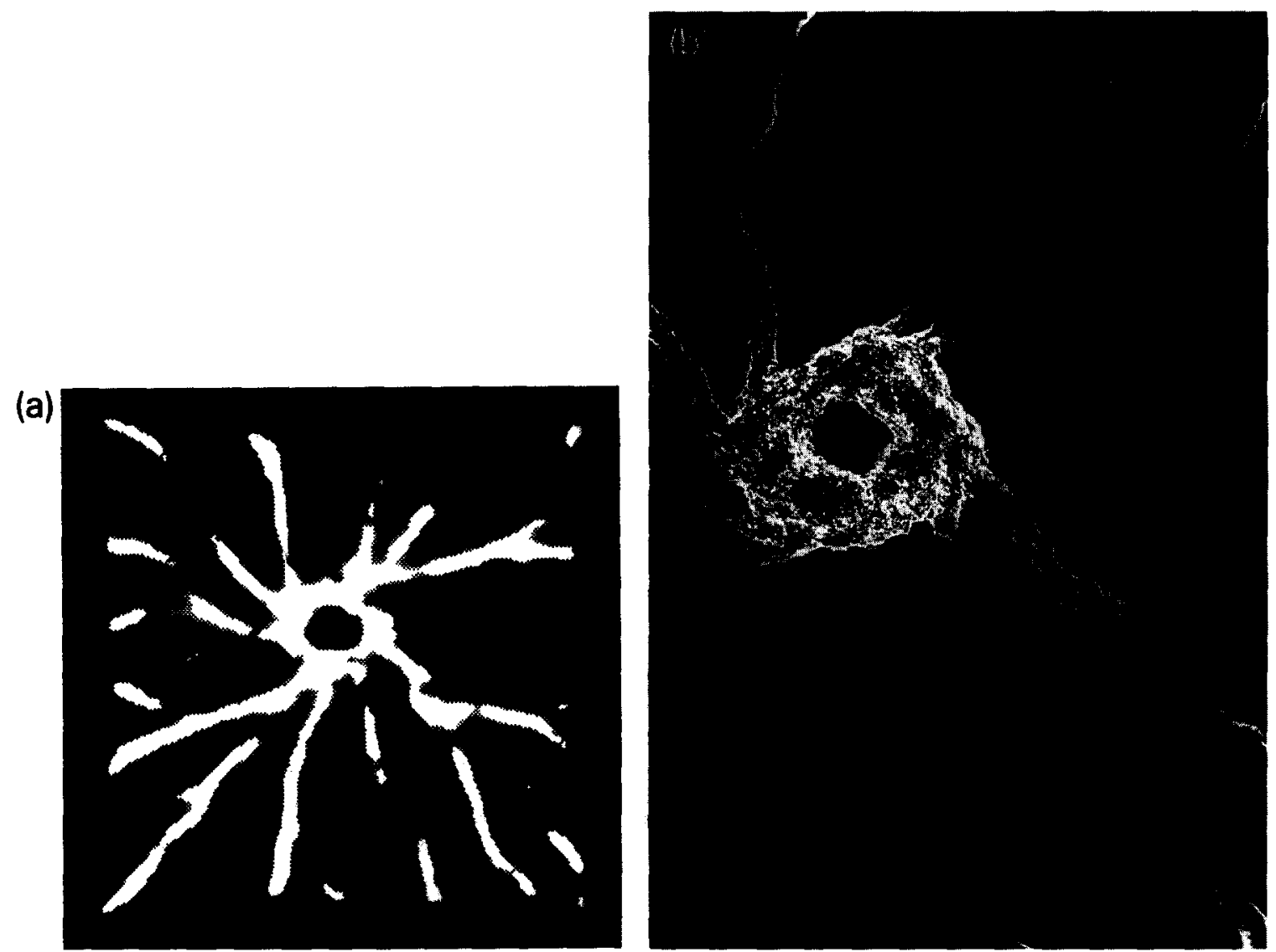

Fig. 9. Stable hole in the core region of the CAMP spiral (a) in a simulation $t=80 \mathrm{~min}$, and (b) in situ (strain NCF; photograph courtesy of P.C. Newell) . Parameter set for (a) as in Table 2 ; in addition the density-dependent cell diffusion coefficient with $\mu_{1}=0.003$, $\mu_{2}=0.009, M=1.2$ and $r=4$ (cf. (5)) was included; initial cell density $0.9 \pm 0.075$. Cell density scale 0.7 (black) -1.7 (white). For numerical scheme see [9].

to a multitude of different problems. An alternative approach to cell aggregation in Dictyostelium employs simulations with discrete particle-like cells with prescribed cAMP-dependent movement rules ([26-28], H.G. Othmer, personal communication).

While the description developed in [12] appears to be valid for Dictyostelium species without periodic chemoattractant waves, such as $D$. minutum and $D$. lacteum, the situation in Dictyostelium discoideum is more complex. Here patterning occurs on two quite distinct spatial scales. First aggregation centres are established as the "sources" of the cAMP waves (autonomously oscillating centres or spiral wave cores developing from disrupted wavefronts) and subsequent aggregation is the consequence of the more or less concentric geometry of the signalling waves coupled with the time-dependent chemotactic cell response, rather than the result of a chemotactic instability. These centres appear to have a characteristic spacing (roughly $1 \mathrm{~cm}$ ), and the mechanism by which this pattern of aggregation territories is specified is unclear. Here we focus on one such aggregation territory and show that there is, in fact, a somewhat different kind of chemotaxis-driven instability present in $D$. discoideum aggregation, which leads to the formation of the cell stream pattern. This patterning process in an ensemble of communicating cells has a number of interesting properties, many of which are quite well reproduced by the proposed minimal model. The instability leading to cell streaming is 
analogous to instabilities found in other biological patterning models (reaction-diffusion and mechano-chemical [29], chemotaxis [12,30,31]); spatial modes associated with a real eigenvalue (in the present case Floquet exponent) go unstable, and the wavenumbers of the unstable modes are essentially determined by the parameters of the governing system (in most cases the dispersion relation has a distinct maximum ${ }^{1}$ ). The route by which the pattern is established, however, is quite different from these "standard" models. The pattern is forced by reaction-diffusion waves, which gives it a distinct polarity (streams perpendicular to the wavefronts rather than isolated humps of cell density). In this sense the cAMP waves act as a dynamic chemical "prepattern", and our model provides a case study for the coupling of two biologically implicated patterning mechanisms, reaction-diffusion and chemotaxis [32].

It is interesting to note that no direct cell-cell interactions are required for the streaming instability. In particular, streaming is also obtained with a density-independent cell diffusivity (numerical simulations, caricature analysis). This is in agreement with the experimental observation that some mutants deficient in cell-adhesion molecules still form streams, albeit less pronounced ones than the aggregation streams of the wildtype ([18], P. Newell, personal communication).

Aggregation via stream formation causes local increase and decrease in chemical excitability to occur in the form of a well-defined spatial structure, which is forced by the excitation waves themselves. In this sense the Dictyostelium aggregation field constitutes an excitable medium with spatio-temporally self-organized inhomogeneous excitability properties. This medium supports spiral wave solutions, which unlike spiral waves in "classical" excitable media have time-dependent geometrical and dynamical properties. In particular, the model relatively accurately reproduces the dependence of the cAMP wavespeed on "wave time" measured experimentally [Fig. 8b]. Gross et al. [24] attribute their observations to speculative biochemical changes which would have to be forced by the cAMP waves. In our model the decrease of the wavespeed occurs with constant parameter values. This underlines that an apparently unexpected phenomenon need not require an intricate biological interpretation, but may arise from relatively few nonlinear interactions. On the other hand, it is conceivable that a slow increase in the excitability of the adenylate cyclase pathway forced by the cAMP waves could also contribute to the observed change in the spiral pattern. It will be interesting to investigate whether results from the classical theory of waves in excitable media [11,33] can be extended to account for the present case of slowly varying (and increasingly anisotropic) excitability. In this context it becomes clear that the linear stability analysis presented here really is a first approach to the problem. It highlights the salient features of the instability mechanism, but a more complete understanding should come from the inclusion of slowly varying excitability, affecting both cAMP wave speed and wavelength. Intuitively, such an extension should also be able to capture the selection of a stream ("stripe") rather than a spot pattern in quantitative terms.

Despite a number of simplifications (most notably the assumption of a continuous cell density), our model of Dictyostelium aggregation, based on biochemical and mechanical mechanisms established on the cellular level, has captured in some detail the cell movement and chemical signalling dynamics observed experimentally. Elaborating on previous theoretical work on cAMP dynamics and the chemotactic response, this study provides a further link between the individual cell properties and the collective patterning modes in this cell ensemble at the onset of multicellular organization.

\footnotetext{
In mechano-chemical models, more complex situations can arise [29].
} 


\section{Acknowledgements}

T.H. and P.K.M. would like to thank P.C. Newell for helpful discussions. T.H. was supported by a graduate studentship from the Boehringer Ingelheim Fonds, the Engineering and Physical Sciences Research Council of Great Britain and a Jowett Senior Scholarship at Balliol College Oxford.

\section{Appendix}

Here we present the Floquet analysis of Eq. (21) and derive conditions for chemotaxis-driven instability in the caricature system $(16)-(19)$.

The monodromy matrix $\boldsymbol{M}$ of (21) can be computed in the following way. System (21) can be solved separately on the $I_{j}^{+}$and $I_{j}^{-}$half-intervals of the $j$ th forcing period, $j \Lambda<\xi<(j+1 / 2) \Lambda$ and $(j+1 / 2) \Lambda<$ $\xi<(j+1) \Lambda$, respectively. The fundamental solutions $\boldsymbol{\Theta}^{ \pm}$are

$$
\boldsymbol{\Theta}^{ \pm}(\xi)=\boldsymbol{Z}^{ \pm} \boldsymbol{\Lambda}^{ \pm}(\xi) \boldsymbol{C}^{ \pm}
$$

where

$$
Z^{ \pm}=\left(z_{1}^{ \pm}, z_{2}^{ \pm}\right), \quad \Lambda^{ \pm}=\left(\begin{array}{cc}
e^{\lambda_{1}^{ \pm} \xi} & 0 \\
0 & e^{\lambda_{2}^{ \pm} \xi}
\end{array}\right),
$$

and the matrices $C^{ \pm}$are constant $2 \times 2$ matrices. Here $\lambda_{1 / 2}^{ \pm}$and $z_{1 / 2}^{ \pm}$denote the eigenvalues and eigenvectors of the constant-coefficient systems corresponding to the $I_{j}^{+}$and $I_{j}^{-}$intervals, respectively. We require continuity of the solution at $\xi=(j+1 / 2) \Lambda$, that is,

$$
\Theta^{+}((j+1 / 2) \Lambda)=\Theta^{-}((j+1 / 2) \Lambda) .
$$

This matching condition yields $\boldsymbol{C}^{-}$,

$$
\boldsymbol{C}^{-}=\left[\boldsymbol{Z}^{-} \boldsymbol{\Lambda}^{-}((j+1 / 2) \Lambda)\right]^{-1} \boldsymbol{Z}^{+} \boldsymbol{\Lambda}^{+}((j+1 / 2) \Lambda) \boldsymbol{C}^{+} .
$$

$\boldsymbol{C}^{-}$is regular if $\boldsymbol{C}^{+}$is chosen to be regular. Hence we have constructed a complete system of linearly independent solutions of (21) for the $j$ th forcing period (with obvious extension for any $\xi$ outside this period; cf. also [34] ). The monodromy matrix, $M=\Theta((j+1) \Lambda) \Theta(j \Lambda)^{-1}$, is then obtained as

$$
\boldsymbol{M}=\left(\boldsymbol{Z}^{+} \boldsymbol{C}^{+}\right)^{-1} \boldsymbol{Z}^{-} \boldsymbol{\Lambda}^{-}((j+1) \boldsymbol{\Lambda}) \boldsymbol{C}^{-} .
$$

The eigenvalues of $\boldsymbol{M}$,

$$
\rho_{1 / 2}=\frac{1}{2}\left[\operatorname{tr} M \pm\left(\operatorname{tr}^{2} M-4 \operatorname{det} M\right)^{1 / 2}\right],
$$

contain the desired information about the stability of the trivial solution of (21).

We carry out this procedure to obtain the dispersion relation in explicit form. For simplicity we take $C^{+}=I$ and $j=0$, and rewrite $\tilde{\mu}=q^{2} \mu$ and $\tilde{\delta}=q^{2} \delta+d n_{0}$. After some algebra, we find from (A.1)-(A.3)

$$
\begin{aligned}
\operatorname{tr} \boldsymbol{M}= & {\left[\left(\lambda_{1}^{+}-\lambda_{2}^{+}\right)(\tilde{\mu}-\tilde{\delta})\right]^{-1}\left[\left(\lambda_{1}^{+}+\tilde{\delta}\right)\left(\lambda_{2}^{+}+\tilde{\mu}\right)\left(e^{\left(\lambda_{1}^{+}-\tilde{\mu}\right) \Lambda / 2}+e^{\left(\lambda_{2}^{+}-\tilde{\delta}\right) \Lambda / 2}\right)\right.} \\
& \left.-\left(\lambda_{1}^{+}+\tilde{\mu}\right)\left(\lambda_{2}^{+}+\tilde{\delta}\right)\left(e^{\left(\lambda_{1}^{+}-\tilde{\delta}\right) \Lambda / 2}+e^{\left(\lambda_{2}^{+}-\tilde{\mu}\right) \Lambda / 2}\right)\right] \\
\operatorname{det} \boldsymbol{M}= & \exp \{-(\tilde{\mu}+\tilde{\delta}) \Lambda\},
\end{aligned}
$$


where

$$
\lambda_{1 / 2}^{+}=\frac{1}{2}\left(-(\tilde{\mu}+\tilde{\delta}) \pm\left[(\tilde{\mu}-\tilde{\delta})^{2}+4 p \bar{\chi} n_{0} q^{2}\right]^{1 / 2}\right)
$$

(note that the eigenvalues for $I^{-}$half-period, $\lambda_{1 / 2}^{-}=-\tilde{\mu},-\tilde{\delta}$ have been incorporated in (A.5),(A.6) directly). For a pattern to grow at least temporarily, we must have $\lambda_{1}^{+}>0$ (since $\lambda_{1 / 2}^{-}$and $\lambda_{2}^{+}$are always negative), i.e. $p \bar{\chi} n_{0} q^{2}>\tilde{\mu} \tilde{\delta}$. In this case it can be shown that $\operatorname{tr} \boldsymbol{M}>0$, and hence the leading Floquet multiplier is $\rho_{1}$. Typical dispersion relations, $\Delta\left(q^{2}\right)=\ln \left(\rho_{1}\right) / \Lambda$, are depicted in Fig. 6. For instability we need $\left|\rho_{1}\right|>1$. Using the fact that $\operatorname{det} M<1$, this can be shown to be equivalent to

$$
\operatorname{tr} \boldsymbol{M}>1+\operatorname{det} \boldsymbol{M} .
$$

Thus instability is associated with a real Floquet multiplier passing through 1. Condition (A.8) constitutes a relationship between the system parameters (including the cAMP wavelength $\Lambda$ ) and the wavelength $q$ of modes in the $y$-direction, and thus provides an explicit criterion for whether a given mode $q$ in the initial perturbation grows or decays [ $\operatorname{tr} \boldsymbol{M}\left(q^{2}\right)>$, or $<, 1+\operatorname{det} \boldsymbol{M}\left(q^{2}\right)$ ]. The actual expression obtained by introducing (A.5)(A.7) into (A.8) is rather cumbersome and will not be discussed here. However, in the limiting cases $A \rightarrow 0$ and $\Lambda \rightarrow \infty$, (A.8) reduces to surprisingly simple relations. Consider first the case $A \rightarrow 0$. We expand both sides of (A.8) with respect to $\Lambda$ about $\Lambda=0$. All terms up to first order cancel, and to order $\Lambda^{2}$, we obtain the result: $\rho_{1}>1$ if

$$
p \bar{\chi} n_{0} q^{2}>2 \tilde{\mu} \tilde{\delta} .
$$

This condition implies that the critical values the product $p \bar{\chi} n_{0}$ must attain to achieve growth in the wavefront $\left(\lambda_{1}^{+}>0\right)$ and overall growth over a full wavelength $\left(\rho_{1}>1\right)$ are simply related by the factor 2 . For $A \rightarrow \infty$ we see immediately from (A.5) that $\rho_{1}>1$ requires $\lambda_{1}^{+}>\min \{\tilde{\mu}, \tilde{\delta}\}$, that is,

$$
p \bar{\chi} n_{0} q^{2}>2\left[\tilde{\mu} \tilde{\delta}+(\min \{\tilde{\mu}, \tilde{\delta}\})^{2}\right],
$$

(note that in this case $\Delta\left(q^{2}\right)$ stays bounded while $\rho_{1}$ diverges). As for our system we have $\tilde{\mu} \ll \tilde{\delta}$, the relations (A.9) and (A.10) are practically identical.

\section{References}

[1] F. Siegert and C.J. Weijer, in: Oscillations and Morphogenesis, ed. L. Rensing (Marcel Dekker, New York, 1993 ) p. 133.

[2] J.J. Tyson, K.A. Alexander, V.S. Manoranjan and J.D. Murray, Physica D 34 (1989) 193.

[3] P.B. Monk and H.G. Othmer, Proc. R. Soc. London B 240 (1990) 555.

[4] P.N. Devreotes, M.J. Potel and S.A. MacKay, Dev. Biol. 96 (1983) 405.

[5] F. Alcantara and M. Monk, J. Gen. Microbiol. 85 (1974) 321.

[6] L. Desbarats, S.K. Brar and C.-H. Sin, J. Cell Sci. 107 (1994) 1705.

[7] T. Höfer, P.K. Maini, J.A. Sherratt, M.A.J. Chaplain, P. Chauvet, D. Metevier, P.C. Montes and J.D. Murray, Appl. Math. Lett. 7 (1994) 1 .

[8] J.-L. Martiel and A. Goldbeter, Biophys. J. 52 (1987) 807.

[9] T. Höfer, J.A. Sherratt and P.K. Maini, Proc. R. Soc. London B 259 (1995) 249.

[10] Y. Tang and H.G. Othmer, Math. Biosci. 120 (1994) 25.

[11] J.J. Tyson and J.P. Keener, Physica D 32 (1988) 327.

[12] E.F. Keller and L.E. Segel, J. Theoret. Biol. 26 (1970) 399.

[13] A. Stevens, Ph.D. Thesis, Heidelberg (1992).

[14] P.R. Fisher, R. Merkl and G. Gerisch, J. Cell Biol. 108 (1989) 973. 
[15] P.C. Newell, G.N. Europe-Finner, G. Liu, B. Gammon and C.A. Wood, in The biology of the chemotactic response, eds. J.P. Armitage and J. Lackie, Symposia of the Society for General Microbiology, Vol. 46 (Cambridge Univ. Press, Cambridge, 1990) p. 273.

[16] D.R. Soll, D. Wessels and A. Sylvester, in: Experimental and Theoretical Advances in Biological Pattern Formation, eds. H.G. Othmer, P.K. Maini and J.D. Murray (Plenum, New York, 1993) p. 325.

[17] P.N. Devreotes and S.H. Zigmond, Annu. Rev. Cell Biol. 4 (1988) 649.

[18] G. Gerisch, J. Cell Sci. 4, Suppl. (1986) 201.

[19] R.T. Tranquillo, S.H. Zigmond and D.A. Lauffenburger, Cell Motil. Cytoskeleton 11 (1988) 1.

[20] J.A. Sherratt, E.H. Sage and J.D. Murray, J. Theor. Biol. 162 (1993) 23.

[21] J.A. Sherratt, Bull. Math. Biol. 56 (1994) 129.

[22] D. Horváth, V. Petrov, S.K. Scott and K. Showalter, J. Chem. Phys. 98 (1993) 6332.

[23] K.W. Morton and D.F. Mayers, Numerical Solution of Partial Differential Equations (Cambridge Univ. Press, Cambridge, 1994).

[24] J.D. Gross, M.J. Peacey and D.J. Trevan, J. Cell Sci. 22 (1976) 645.

[25] A.T. Winfree, Physica D 49 (1991) 125.

[26] H. Parnas and L.E. Segel, J. Cell Sci. 25 (1977) 191.

[27] S.A. MacKay, J. Cell Sci. 33 (1978) 1.

[28] D.A. Kessler and H. Levine, Phys. Rev. E 48 (1993) 4801.

[29] J.D. Murray, Mathematical Biology (Springer, Berlin, 1989).

[30] P.K. Maini, M.R. Myerscough, K.H. Winters and J.D. Murray, Bull. Math. Biol. 53 (1991) 701.

[31] M. Mimura, T. Tsujikawa, R. Kobayashi and D. Ueyama, Forma 8 (1993) 179-195.

[32] B.C. Goodwin, S.A. Kauffman and J.D. Murray, J. theor. Biol. 163 (1993) 135-144.

[33] A.S. Mikhailov, V.A. Davydov and V.S. Zykov, Physica D 70 (1994) 1.

[34] J.A. Sherratt, Eur. J. Appl. Math. (1995), in press.

[35] M. Dworkin and K.H. Keller, J. Biol. Chem. 252 (1977) 864. 